\title{
Nonlinearly Realized Gauge Theories for LHC Physics
}

\section{Daniele Binosi}

European Centre for Theoretical Studies in Nuclear Physics and Related Areas (ECT*) and Fondazione Bruno Kessler, Trento, Italy

E-mail: binosidect.it

\section{Daniele Bettinelli, Andrea Quadri*}

Univ. di Milano and INFN, Sez. di Milano, Italy

E-mail: andrea.quadri@mi.infn.it

\begin{abstract}
We consider a minimal nonlinearly realized electroweak theory where mass generation happens à la Stückelberg. Deformation of the nonlinearly realized gauge symmetry is controlled by functional methods. The Weak Power Counting allows to select uniquely the Hopf algebra of the theory and gives definite predictions on the Beyond-the-Standard Model (BSM) sector of the theory: the latter includes one CP-odd and two charged physical scalars (in addition to the Higgs-like CP-even resonance). The model interpolates between a purely Stückelberg and a Higgs scenario. It can be used in order to check whether the presence of a Stückelberg mass component can already be excluded on the basis of the existing LHC7-8 data.
\end{abstract}

The European Physical Society Conference on High Energy Physics

18-24 July, 2013

Stockholm, Sweden

${ }^{*}$ Speaker. 
The LHC discovery of a new physical scalar resonance of mass about $125 \mathrm{GeV}$ has opened the way to the experimental verification of the electroweak Spontaneous Symmetry Breaking (SSB) mechanism realized in Nature.

The favourite candidate after the LHC7-8 data is the Standard Model (SM) Higgs mechanism. It is therefore timely to check whether one can already exclude, on the basis of the current LHC data, one of the most ancient competitors to the SSB à la Higgs, namely the Stückelberg mechanism [1]. The latter allows to introduce gauge-invariant mass terms for the gauge bosons and matter fermions by using an operatorial gauge transformation controlled by a group element $\Omega \in \mathrm{SU}(2)$, parameterized by the independent coordinates $\phi_{a}$, in a way preserving physical unitarity [2]. The gauge symmetry is nonlinearly realized, as a consequence of the nonlinear SU(2) constraint.

Nonlinearity is reflected into the severe UV divergences of the theory. Since the discovery of the Local Functional Equation (LFE) [3], it has been known that 1-PI amplitudes involving at least one $\phi_{a}$-leg (descendant amplitudes) are uniquely fixed in terms of amplitudes with no external $\phi_{a^{-}}$ legs (ancestor amplitudes). The LFE controls the deformation of the classical nonlinearly realized gauge symmetry, induced by radiative corrections.

Moreover, while there are infinitely many divergent descendant amplitudes already at one loop level, the number of divergent ancestor amplitudes is finite at each loop order, provided that the Weak Power Counting (WPC) holds [4].

The WPC selects uniquely the Hopf algebra of the theory. The number of UV divergent ancestor amplitudes increases order by order in the loop number and thus the model is not renormalizable by power-counting. The implementation of the program based on the LFE and the WPC has been developed in Yang-Mills theory for $\mathrm{SU}(2)$ in [5] and for the semisimple group $\mathrm{SU}(2) \times \mathrm{U}(1)$ in [6]. The electroweak model with physical scalar resonances has been studied in [7].

Since these theories are not power-counting renormalizable, their UV-completion (if any) is not known. Therefore they can be used as an effective low-energy description, valid up to a certain energy scale $Q$. Loosely speaking, one expects that this is the scale where violation of unitarity occurs in physical scattering amplitudes.

Interestingly enough, in the nonlinearly realized electroweak gauge theory in the presence of physical scalar resonances [7], $Q$ can be pushed at arbitrarily high energy, if the parameters of the theory are fine-tuned towards the SM-like region.

The inclusion of a single physical scalar resonance in the nonlinearly realized electroweak model is not possible if the WPC is to hold. The minimal choice is a SU(2) doublet of fields [7] $\chi=\chi_{0}+i \chi_{a} \tau_{a}$. Thus there is also a mass invariant generated as in the usual Higgs mechanism from the $S U(2)$ doublet of scalars $\chi$ :

$$
\mathscr{L}_{\text {mass,linear }}=\frac{1}{4} \operatorname{Tr}\left(D_{\mu} \chi\right)^{\dagger} D^{\mu} \chi .
$$

$\chi_{0}$ acquires a vacuum expectation value $v$, so that it is split according to $\chi_{0}=v+X_{0}$. SSB, triggered by a suitable quartic potential, must occur for the $S U(2)$ doublet along the $\chi_{0}$-component. The reason is that otherwise one cannot accommodate for the suppression of the decay width of $X_{0} \rightarrow \gamma \gamma$ with respect to the decay modes $X_{0} \rightarrow V V, V=W, Z$ (which, without SSB, would be radiatively generated as well). The masses of the $W$ and $Z$ bosons are thus given by

$$
M_{W}=\frac{g v}{2} \sqrt{1+A \frac{f^{2}}{v^{2}}}, \quad M_{Z}=\frac{G v}{2} \sqrt{1+\frac{f^{2}}{v^{2}}\left(A+\frac{B f^{2}}{2}\right)} .
$$


$g, g^{\prime}$ are the $\mathrm{SU}(2)$ and hypercharge $\mathrm{U}(1)$ coupling constants respectively and $G=\sqrt{g^{2}+g^{\prime 2}}$.

The parameters $A, B$ describe the contribution to the gauge boson masses induced by the Stuickelberg mechanism. In particular $B$ controls the violation of custodial symmetry in the gauge boson sector. Such a violation is a characteristic feature of nonlinearly realized theories based on the WPC [8]. If $A=0, B=0$ one gets back the SM scenario where the electroweak SSB is realized through the linear Higgs mechanism. In this case the $\phi_{a}$ decouple and the Goldstone bosons are to be identified with the $\chi_{a}$ fields. At $B=0$ and $A \neq 0$ a fraction of the mass of the gauge bosons is generated via the Stückelberg mechanism while still preserving the Weinberg relation. Finally, $A \neq 0$ and $B \neq 0$ corresponds to the most general Stückelberg case with two independent mass terms for the $W$ and the $Z$ bosons.

The theory makes definite predictions on the field content of the BSM sector: there must be four scalar resonances, two charged ones and two neutral ones, one CP-even (to be eventually identified with the $125 \mathrm{GeV}$ resonance recently discovered at LHC) and one CP-odd.

Current LHC data clearly favour a scenario where new physics contributions, resulting in deviations from the SM values, are small [9]. The physically interesting case is therefore achieved in the small $A$ limit at $B=0[10]$. We remark that if even a fraction of the gauge boson masses is generated by the Stückelberg mechanism, tree-level unitarity in the scattering of longitudinally polarized $\mathrm{W}$ bosons is violated, despite the exchange of a physical scalar resonance. This feature is a characteristic footprint of nonlinearly realized theories.

\section{References}

[1] see e.g. H. Ruegg and M. Ruiz-Altaba, Int. J. Mod. Phys. A 19 (2004) 3265 [hep-th/0304245].

[2] R. Ferrari and A. Quadri, JHEP 0411 (2004) 019 [hep-th/0408168].

[3] R. Ferrari, JHEP 0508 (2005) 048 [hep-th/0504023]; D. Bettinelli, R. Ferrari and A. Quadri, JHEP 0703 (2007) 065 [hep-th/0701212].

[4] D. Bettinelli, R. Ferrari and A. Quadri, Int. J. Mod. Phys. A 23 (2008) 211 [hep-th/0701197]; R. Ferrari and A. Quadri, Int. J. Theor. Phys. 45 (2006) 2497 [hep-th/0506220].

[5] D. Bettinelli, R. Ferrari and A. Quadri, Phys. Rev. D 77 (2008) 045021 [arXiv:0705.2339 [hep-th]]; D. Bettinelli, R. Ferrari and A. Quadri, Phys. Rev. D 77 (2008) 105012 [Erratum-ibid. D 85 (2012) 129901] [arXiv:0709.0644 [hep-th]]; D. Bettinelli, R. Ferrari and A. Quadri, J. General. Lie Theor. Appl. 2 (2008) 122 [arXiv:0712.1410 [hep-th]].

[6] D. Bettinelli, R. Ferrari and A. Quadri, Int. J. Mod. Phys. A 24 (2009) 2639 [Erratum-ibid. A 27 (2012) 1292004] [arXiv:0807.3882 [hep-ph]]; D. Bettinelli, R. Ferrari and A. Quadri, Acta Phys. Polon. B 41 (2010) 597 [Erratum-ibid. B 43 (2012) 483] [arXiv:0809.1994 [hep-th]]; D. Bettinelli, R. Ferrari and A. Quadri, Phys. Rev. D 79 (2009) 125028 [Erratum-ibid. D 85 (2012) 049903] [arXiv:0903.0281 [hep-th]].

[7] D. Binosi and A. Quadri, JHEP 1302 (2013) 020 [arXiv:1210.2637 [hep-ph]].

[8] A. Quadri, Eur. Phys. J. C 70 (2010) 479 [arXiv:1007.4078 [hep-th]].

[9] P. P. Giardino, K. Kannike, I. Masina, M. Raidal and A. Strumia, arXiv:1303.3570 [hep-ph]; J. Ellis and T. You, JHEP 1306 (2013) 103 [arXiv:1303.3879 [hep-ph]].

[10] D. Bettinelli and A. Quadri, arXiv:1307.2420 [hep-ph]. 